Questions vives

\section{Questions Vives}

Recherches en éducation

$N^{\circ} 28$ | 2017

De l'indifférenciation à la différenciation

\title{
Devenir enseignant-chercheur à l'heure de l'autonomie des universités. Une recherche sur les représentations du métier chez les doctorants
}

To become a teacher-researcher at the time of university autonomy. A research on representations of the profession among PhD students

\section{Charlyne Millet et Emmanuelle Annoot}

\section{OpenEdition \\ Journals}

Édition électronique

URL : http://journals.openedition.org/questionsvives/2547

DOI : 10.4000/questionsvives.2547

ISSN : $1775-433 X$

\section{Éditeur}

Université Aix-Marseille (AMU)

Édition imprimée

Date de publication : 29 décembre 2017

ISBN : 978-2-912643-52-0

ISSN : 1635-4079

Référence électronique

Charlyne Millet et Emmanuelle Annoot, « Devenir enseignant-chercheur à I'heure de l'autonomie des universités. Une recherche sur les représentations du métier chez les doctorants », Questions Vives [En ligne], № 28 | 2017, mis en ligne le 15 novembre 2018, consulté le 30 avril 2019. URL : http:// journals.openedition.org/questionsvives/2547; DOI : 10.4000/questionsvives.2547

Ce document a été généré automatiquement le 30 avril 2019.

\section{(†)

Questions Vives est mis à disposition selon les termes de la licence Creative Commons Attribution Pas d'Utilisation Commerciale - Pas de Modification 4.0 International. 


\section{Devenir enseignant-chercheur à l'heure de l'autonomie des universités. Une recherche sur les représentations du métier chez les doctorants}

To become a teacher-researcher at the time of university autonomy. A research on representations of the profession among $\mathrm{PhD}$ students

Charlyne Millet et Emmanuelle Annoot

\section{Introduction : une recherche sur les doctorants exerçant une mission d'enseignement}

1 La question de la transformation des pratiques pédagogiques des enseignants-chercheurs s'inscrit dans un projet politique qui vise une amélioration de la réussite des étudiants (Bertrand, 2014). Or, les recherches sur les enseignants du supérieur et leur métier sont rares (Millet, Oget \& Sonntag, 2016) et celles qui sont consacrées aux doctorants qui se destinent à une carrière académique encore plus. En effet, dans un contexte marqué par une « précarité récente », Delphine Serre (2015) constate que « la situation des doctorants est principalement étudiée au prisme de la question de leur avenir et de leur insertion professionnelle ». Depuis l'harmonisation européenne des diplômes, l'auteure constate le poids de contraintes organisatrices de plus en plus fortes s'appuyant davantage sur des critères gestionnaires que sur des critères disciplinaires pour tendre à des formes de standardisation du parcours doctoral : le doctorat « ne se limite plus à la rédaction d'une thèse de qualité mais implique des publications, des communications et de multiples engagements dans l'enseignement, les tâches collectives, l'animation de la recherche " (Serre, 2015). Développer des recherches en sciences de l'éducation en étudiant les 
représentations des doctorants sur le métier d'enseignant-chercheur à partir de leurs premières expériences d'enseignement à l'université constitue un enjeu pour comprendre la manière dont ils orienteront leur action (Lameul, 2008). Nous situons notre recherche dans la continuité d'un programme $e^{1}$ plus vaste dirigé par David Adé (CETAPS, université de Rouen) et Thierry Piot (CERSE, université de Caen) qui a conduit à la publication d'un ouvrage collectif (Adé \& Piot, 2018). Une attention particulière aux enseignantschercheurs qui entrent dans le métier, c'est-à-dire qui exercent leur activité «en responsabilité » et avec une ancienneté inférieure à cinq ans, indépendamment du statut, et sans délégation ni aide possible, y a été portée. Cet article a pour but d'entreprendre une réflexion sur l'entrée potentielle dans le métier en mettant en regard des recommandations nationales qui misent sur l'innovation pédagogique pour améliorer la qualité de l'enseignement supérieur et les représentations du métier d'enseignant à l'université chez des doctorants ayant une première expérience d'enseignement. Notre recherche interroge l'impact du contexte universitaire sur la construction des identités professionnelles des doctorants susceptibles d'entrer dans le métier d'enseignantchercheur. Nous allons dans un premier temps présenter les transformations récentes du métier d'enseignant-chercheur, en nous appuyant successivement sur les travaux de Faure, Soulié et Millet (2006), Dahan et Mangematin (2010) et Fave-Bonnet (2002). Puis, nous présenterons les axes heuristiques de notre recherche, la méthodologie du recueil des données avant d'apporter des éléments d'interprétation et nos premières conclusions .

\section{Un contexte en tension}

2 Au sein de l'enseignement supérieur français, l'université a connu une succession de réformes depuis l'instauration du processus de Bologne à partir des années 2000, à laquelle a succédé la mise en place de la LRU (loi relative aux libertés et aux responsabilités des universités) à partir de 2007. Les effets du passage à l'autonomie des établissements sur les pratiques pédagogiques des universitaires ont été peu étudiés. Depuis le rapport Espéret (2001) jusqu'à aujourd'hui, le métier d'enseignant-chercheur a connu une série de transformations non seulement dues à ces changements structurels mais aussi à l'accueil de nouveaux publics étudiants tant en formation initiale que continue.

\subsection{L'accroissement du travail administratif}

3 Sylvia Faure, Charles Soulié et Mathias Millet, dans leur article consacré au travail des enseignants-chercheurs, paru en 2006 dans la revue Regards sociologiques, dénoncent, dans un contexte de massification/diversification des publics étudiants et de rationalisation du fonctionnement du monde académique et scientifique, l'accroissement du temps de travail administratif. Les auteur-e-s évoquent le jeu de concurrence dans lequel la profession universitaire est entrée. On peut dire que :

L'université est entrée dans un processus de mutation accéléré. [...] Mais par-delà ces différences, un même constat relatif au métier des enseignants chercheurs s'impose. C'est celui d'une rationalisation, bureaucratisation croissante de leur travail en raison notamment de la massification, diversification des publics étudiants, de la complexification des procédures de financements de la recherche, comme des réformes successives de l'enseignement supérieur et de la recherche. 
Lesquelles réformes s'enchaînent rapidement les unes aux autres et finissent par produire chez nombre d'académiques un sentiment croissant d'impuissance, comme de dépossession généralisée (Faure, Soulié \& Millet, 2006, pp. 137-138).

Ces mutations semblent induire des changements identitaires, tantôt interprétés comme une crise de l'« ethos académique » (Faure, Soulié \& Millet, 2006) ou comme la promesse de reconfigurations de la profession d'universitaire (Dahan \& Mangematin, 2010 ; FaveBonnet, 2002).

\subsection{Le rapport au temps révélateur des mutations des identités professionnelles}

4 L'augmentation des effectifs étudiants et la transformation de l'architecture des formations universitaires selon des critères de qualité prédéfinis ont introduit des modifications du travail pédagogique pour les enseignants-chercheurs. La LRU mise en place en 2007 amenant des formes d'autonomie budgétaire dans les universités a contraint les universitaires à faire face à des charges administratives nouvelles. Aubépine Dahan et Vincent Mangematin (2010, p.15) en apportent quelques illustrations: la recherche de financements privés d'origine industrielle à la suite de la réduction des budgets publics, la démultiplication des positions administratives (par exemple, les responsabilités de diplôme ou de parcours) causée par la complexification de l'offre universitaire, les activités liées à la professionnalisation des études universitaires, etc. Ces reconfigurations peuvent se lire au prisme de la question temporelle. Dahan et Mangematin (2010) indiquent que les professions doivent faire face aux exigences grandissantes de «la forme organisationnelle ». La question du temps de travail alloué à la gestion des tâches administratives à l'université est d'ailleurs au centre des " complaintes récurrentes" et critiques relevées dans les entretiens d'universitaires recueillis par les auteur-e-s. L'idée principale exprimée par les auteur-e-s est que l'invocation de cette "perte de temps" peut constituer "un fil rouge révélateur de l'image que les universitaires se font de leur profession, de ce qu'il est légitime ou non de faire» (p.15). Les auteur-e-s évoquent l'idée d'une déstabilisation de leur logique professionnelle «dont l'objet premier est de remplir une mission, déstabilisée par la logique de l'organisation, dont l'objet premier est sa propre survie » (p. 16).

Pour les universitaires, il y a nécessité d'opérer une "trajectoire d'adaptation identitaire » qui permettrait alors de faire correspondre «ce qu'ils font avec ce qu'ils sont », pour reprendre la formule de Pratt et al. (2006) sur la construction de l'identité professionnelle (Dahan et Mangematin, 2010, p. 16). Saisir ce que sont les universitaires, c'est justement s'interroger sur leur identité professionnelle, laquelle, conceptualisée par Claude Dubar (1998) en sociologie des professions, désigne les éléments symboliques ou matériels par lesquels les membres d'une profession se définissent (Dahan et Mangematin, 2010, p. 16). Or, si nous admettons que les missions évoluent, les identités également. Ces identités professionnelles en mutation peuvent alors se traduire par des "parcours" de carrière différenciés selon les activités valorisées (Millet, 2011). Les activités de recherche (par exemple, lectures ou travail à la paillasse) sont considérées comme légitimes mais ne bénéficient pas d'un temps suffisant. Les activités périphériques sont quant à elle vécues comme chronophages et peu valorisées. Nous sommes là au centre de la question de l'organisation des bureaucraties professionnelles et de l'identité professionnelle des enseignants-chercheurs : « Il parait donc essentiel de comprendre les représentations que la profession se fait d'elle-même pour renouveler le débat 
professions/organisations» (Dahan et Mangematin, 2010, p. 23). La recherche que nous avons entreprise s'inscrit dans cette perspective.

\subsection{Un sentiment de dégradation du métier d'universitaire}

6 Les transformations et l'élargissement des missions des universitaires sont lisibles depuis l'instauration de la loi Savary du 26 janvier 1984. Ces transformations impliquent des changements dans les termes mêmes employés pour désigner la profession. Du terme "professeur", nous sommes passés au terme "enseignant-chercheur ». Les tâches d'enseignement se sont également accrues, liées à l'augmentation des effectifs étudiants. Les pratiques pédagogiques ont également évolué (des cours magistraux aux travaux dirigés). En outre, le morcellement des cursus et la semestrialisation ont augmenté la charge de travail. Pour Marie-Françoise Fave-Bonnet (2002, p.34), «cette situation entraine une crispation sur le nombre d'heures d'enseignement au détriment du contenu et des pratiques». Ce qui se développe, c'est une activité d'enseignement «sans formation pédagogique, sans évaluation, sans valorisation, sans lien avec la recherche, [où] l'enseignement à l'Université devient une activité routinière ". Les changements dans la recherche sont également très marqués : dans le temps (compétitivité), dans l'espace, dans les modes de travail également. En outre, Fave-Bonnet remarque que le développement de divers organismes de recherche (CNRS, INSERM, CEA) ne fait qu'accroître la séparation institutionnelle entre université et recherche. À cela s'ajoutent les missions liées aux responsabilités et fonctions administratives «dans une tradition universitaire où le patriotisme d'université est faible et peu récompensé » (Bourdieu, 1984, cité par Fave-Bonnet, 2002, p. 36) (entendu en matière de carrière). C'est une crise $\mathrm{du}$ modèle sur lequel s'est construite l'université française: «la situation que nous venons d'esquisser, et l'impossibilité d'assumer des missions multiples et parfois contradictoires, a engendré un bouleversement des valeurs sur lesquelles se fondait la profession" (p. 37). Marie-Françoise Fave-Bonnet insiste sur l'idée qu'il s'agit d'une profession éclatée en de multiples métiers, une profession axée sur sa spécialité :

L'identification principale est celle de l'individu à sa réputation au sein de la communauté disciplinaire. C'est la reconnaissance par les pairs qui est recherchée avant tout, et l'engagement professionnel est fortement conditionné par l'espoir d'un accroissement de cette reconnaissance souvent enracinée dans une conception de la vocation. La stratégie professionnelle est celle de l'accumulation des savoirs et de la lutte pour le maintien de la rareté de la formation (pp. 37-38).

L'auteure met en avant les différences dans les carrières qui varient en fonction des investissements différenciés, selon la discipline, l'âge, le sexe :

7 Les investissements personnels dans les différentes tâches sont essentiellement le statut (qui détermine l'ampleur des responsabilités et la dimension du réseau scientifique), la discipline (qui structure les modalités et les lieux d'exercice de la recherche) et l'étape de la carrière (p. 39).

8 Ce à quoi nous ajouterions la localisation géographique et le moment du recrutement. Enfin, ces changements dans les valeurs professionnelles, caractérisés par des rapports hiérarchiques en mutation, en déplacement («déconditionnement à l'autorité professorale », p. 40), par une gestion du temps de travail complexe et un rapport à l'argent ambivalent. In fine, c'est un sentiment de perte de prestige social qui englobe la profession universitaire. 
9 En conclusion, un sentiment de dégradation du métier émane des universitaires, ce que nous avons par ailleurs souligné dans notre article (Millet, Oget \& Sonntag, 2016) comme à l'origine d'un besoin de reconnaissance de la part de ces professionnels. Au sein d'une réflexion portant sur les transformations qui caractérisent l'enseignement supérieur ces dernières années, nous envisageons le cas spécifique des doctorants découvrant le métier d'enseignant-chercheur. Pour notre recherche, souhaitant pallier le manque de publications sur ce sujet, nous nous sommes intéressées aux discours présents, de cette nouvelle génération de chercheurs, universitaires en devenir ou aspirants à l'être.

\title{
2. Deux axes heuristiques orientant l'entrée dans le métier : la matrice disciplinaire et la relation aux pairs
}

10 Durant leur parcours doctoral, certains étudiants exercent des missions d'enseignement en qualité de contractuels de l'enseignement supérieur, en occupant une fonction d'attaché temporaire d'enseignement et de recherche (ATER), d'enseignant vacataire ou d'allocataire bénéficiant d'un contrat doctoral inauguré en 2009 après la disparition des Centres d'initiation à l'enseignement supérieur (CIES). Grâce au recueil de nos données, nous avons souhaité accéder aux représentations du métier qu'ont ces doctorants, saisir comment elles émergent et comment elles se façonnent au cours de leur expérience doctorale. Plus précisément, nous avons cherché à étudier le lien entre deux composantes fondamentales du métier: l'enseignement et la recherche dans leur discipline de référence, considérée comme élément clé de leur construction identitaire (Faure, Soulié \& Millet, 2008), à partir de ce que les doctorants évoquent de leurs pratiques d'enseignement. En effet, Faure, Soulié et Millet (2008) formulent l'hypothèse que :

\begin{abstract}
La « matrice disciplinaire » est supposée orienter de manière significative les modes d'entrée dans le métier mais aussi les pratiques des maîtres de conférences et des professeurs d'université, et, de fait, leurs manières de concevoir leur métier, d'organiser leur temps de travail, de supporter plus ou moins bien les contraintes institutionnelles et administratives, dans un contexte de massification des publics étudiants (très hétérogènes sur le plan scolaire), de réformes sur l'organisation des études (LMD), et de mise en concurrence des filières (aboutissant à une multiplication des diplômes) (pp. 79-80).
\end{abstract}

11 À la suite de la recherche sur les enseignants-chercheurs débutants (Etienne \& Annoot, 2014), nous avons formulé l'hypothèse de l'influence première des pairs dans l'apprentissage du métier d'enseignant à l'université à travers des formes de compagnonnage (Paul, 2009, p. 94) : « la philosophie du compagnonnage réside dans cette vieille constante à ne pas dissocier les trois composantes de sa mission: apprendre, pratiquer et transmettre ». En appui sur ces axes heuristiques propres à notre recherche, nous avons conçu un questionnaire accessible à partir d'un lien Internet sur un espace protégé et diffusé grâce à l'accord des directeurs de différentes Écoles doctorales d'une communauté d'universités et d'établissements (COMUE) située en province française, au cours des mois de mars 2016 et février 2017. Par ce protocole spécifique, l'accès aux données personnelles des enquêtés par les enquêtrices était exclu. 


\section{La méthodologie et l'outil du recueil de données}

12 Un questionnaire divisé en quatre parties et composé d'une quarantaine de questions à choix multiple et ouvert a été diffusé par voie électronique à l'ensemble des doctorants en sciences humaines et sociales, droit économie gestion, sciences et santé, lettres et langues, assurant une mission d'enseignement durant la période du $1^{\mathrm{er}}$ au 30 mars 2016 et au mois de février 2017. Dans un premier temps, nous avons questionné le parcours doctoral de ces étudiants, les conditions de préparation de leur thèse (année d'inscription, financement, discipline, encadrement, etc.) et nous les avons interrogés également sur leurs motivations à s'inscrire en doctorat. Notre recherche s'est centrée par la suite sur les activités d'enseignement et l'organisation du travail doctoral : quels sont le volume horaire et le niveau des enseignements effectués? Quels sont les obstacles rencontrés dans les activités d'enseignement et quels sont les soutiens de collègues enseignants-chercheurs dont les doctorants ont pu bénéficier? Puis nous avons porté notre attention sur les représentations du métier d'enseignant-chercheur en invitant les doctorants à s'interroger sur ce qui compose, selon eux, ce métier, ses dimensions, ses différentes activités et les liens qu'ils ont établis entre l'enseignement et la recherche. Nous avons interrogé également les modalités d'organisation de leur temps de travail avant de poursuivre dans une avant-dernière partie sur les perspectives professionnelles envisagées et l'évolution de leur projet professionnel au cours de leur doctorat. Une dernière partie, plus formelle, visait à recueillir les informations classiques et nécessaires au traitement des données de l'enquête, à savoir et de façon non exhaustive, le sexe, l'âge, la situation familiale ou encore les catégories socioprofessionnelles de l'entourage parental des enquêtés. Nous avons souhaité également savoir si les enquêtés détenaient un concours de l'enseignement primaire ou secondaire. Ce questionnaire a été établi et diffusé avec le logiciel Sphinx Online. La majorité des répondants sont des femmes, nées entre 1981 et 1988, célibataires et sans enfants et n'ayant pas d'autre activité que celle liée au doctorat.

En résumé, notre enquête a eu pour objectif de comprendre comment cette génération de doctorants appréhendait les dimensions du métier d'universitaire à l'heure de l'autonomie des universités. Existe-t-il une spécificité du rapport au métier de cette génération de futurs enseignants-chercheurs? En interrogeant leurs représentations, nous souhaitions faire émerger les bases d'une identité professionnelle et d'une identité de métier en construction (Fave-Bonnet, 2002) et les rapports au travail et au métier que les mutations de l'université ont pu induire depuis plusieurs décennies.

\section{Analyse thématique des résultats}

Nous avons obtenu une quarantaine de réponses (41) à notre questionnaire. Celui-ci avait une visée exploratoire et n'avait pas d'objectif de représentativité. Nos résultats nous servent et nous serviront de support à l'élaboration d'une recherche que nous espérons de plus grande ampleur. Pour cet article, nous avons choisi de centrer la présentation de nos résultats sur les questions portant sur les représentations professionnelles et sur celles portant sur les poursuites professionnelles. Avant cela, nous allons également présenter l'ensemble des motifs qui participent à l'inscription en thèse de doctorat pour 
cette population de doctorants. L'analyse des réponses aux autres questions a donc sciemment été mise de côté.

\subsection{Les motivations à s'inscrire en doctorat}

15 Dans un premier temps, nous avons cherché à déterminer quelles avaient été les motivations à s'inscrire en doctorat. L'analyse thématique des résultats met au jour plusieurs ensembles de motifs qui concourent à cette inscription. Le souhait précis de devenir enseignant-chercheur est évoqué seulement par cinq personnes. Pour les autres, c'est avant tout le désir de « faire de la recherche » qui l'emporte dans les motivations. Il s'agit aussi pour certaines personnes "d'achever les études universitaires en allant jusqu'au bout, en validant le diplôme terminal ». L'inscription en doctorat correspond ainsi à « une suite logique après le master recherche ». Pour cinq autres personnes, c'est aussi la concrétisation d'un " projet à long terme », parfois « clairement présent depuis la licence ». En outre, l'aspect intellectuel des études de doctorat ressurgit régulièrement dans les verbatim, associé aux termes "enrichissement ", "passion», "curiosité». Le doctorat est même présenté comme une «aventure scientifique » où il est possible « de rencontrer des personnes intéressantes». À ces motifs s'ajoute un ensemble de conditions, financières notamment, c'est-à-dire et par exemple, nous citons, «la proposition d'un contrat sur trois ans avec financement" ou "l'obtention d'une allocation doctorale » et s'ajoutent également un ensemble d'influences positives du milieu universitaire qui amène l'étudiant à s'inscrire en doctorat. Une doctorante évoque "les échos absolument positifs durant [sa] soutenance de mémoire de master 2 : cela m'a donné confiance en moi, car j'ai été comme "invitée" à m'inscrire en doctorat ». Il s'agit pour d'autres cas du soutien des personnes qui allaient diriger la thèse. Nous pouvons à ce propos souligner que $62,1 \%$ des répondants à notre questionnaire étaient inscrits dans la même université que celle dans laquelle ils sont inscrits aujourd'hui en thèse.

\subsection{Les dimensions du métier d'enseignant-chercheur}

16 Venons-en désormais aux dimensions du métier. À la question « pouvez-vous définir les dimensions du métier d'enseignant-chercheur aujourd'hui ?", les réponses sont de trois ordres. Certains doctorants répondent par l'investissement conjoint dans trois activités que sont la recherche, l'enseignement et l'administration: «Je crois: une partie recherche (travail individuel, travail d'équipe, travail de valorisation des travaux sous forme de publications, de communications...) ; une partie enseignement et encadrement (cours, TD, suivi des M2 voire des thèses) ; une partie plus administrative (responsabilité de certaines années d'enseignement, mise au point des maquettes, responsabilité au sein du laboratoire voire dans les instances de la Fac).» D'autres évoquent exclusivement les dimensions recherche et enseignement en explicitant le lien qui les unit. Pour ces doctorants, l'enseignement et la recherche sont clairement «imbriqués", " complémentaires", nous citons, «la recherche sert la formation, la formation doit s'appuyer sur la recherche ", ou " la recherche est le portail vers la formation ", dans un «va-et-vient théorie-pratique, gage d'un enseignement de qualité ». La transmission du savoir, la formation des étudiants, le développement de leur esprit critique ou leur réussite universitaire et leur orientation sont ainsi évoqués. La valorisation et la transmission de la recherche sont également explicitées, ainsi que le rayonnement universitaire. La recherche de «crédits, de contrats, de financements » est également 
bien connue tout comme les activités de direction. Nous constatons pour certains une valorisation des activités de recherche aux dépens des activités administratives bien que la question n'incitait pas à hiérarchiser la valeur des tâches: "L'activité la plus chronophage du métier est sans doute l'administration. Les activités de recherche (discussions formelles ou informelles avec d'autres chercheurs, échange d'idées, conférences, séminaires, colloques, articles) représentent sans doute la part la plus passionnante du métier. » Pour une personne, le métier est difficilement définissable et est qualifié de "sur-mesure", c'est-à-dire que «les dimensions varient selon les préférences des personnes».

\subsection{La découverte progressive du métier}

Si leur première expérience d'enseignement leur a permis d'appréhender les dimensions du métier, elle a aussi contribué à modifier leurs représentations sur le métier. À la question « votre expérience d'enseignement vous permet-elle de vous projeter dans le métier d'enseignant-chercheur?», 80,8\% des répondants l'affirment de façon positive. Pour 85,7 \% de ces répondants affirmatifs, la vision du métier a évolué depuis l'inscription en thèse de doctorat.

\subsubsection{Le temps consacré à la recherche concurrencé par l'exercice d'autres activités}

18 Ce qui est mis unanimement en cause, c'est la place prépondérante du temps consacré à l'enseignement et aux activités administratives au détriment de la place de la recherche, ce qui n'était pas imaginé avant l'inscription en thèse. Ainsi, le «couplage entre enseignement et recherche [est] difficile (particulièrement au début de carrière car la préparation des cours demande beaucoup de temps) donc la recherche est au second plan dans ces moments. Pour la recherche, [il y a] beaucoup d'administratif : besoin de chercher des crédits, contrats, financements... ». Le " temps réservé à l'enseignement » est mis en avant. Les témoignages sur les premières expériences d'enseignement dénotent une vision plutôt positive de l'activité. L'expérience d'enseignement est porteuse de «confiance en soi », « d'enrichissement personnel » et de «plaisir [lié à] la transmission ", à la "pédagogie ». Nous soulignons que le couple formation/recherche qui caractérise l'université française a été intégré par la plupart de ces doctorants. Enseigner correspond à une activité de diffusion des connaissances : « je nourris les cours que je dispense par mes "découvertes" ", "le chercheur participe à la diffusion des connaissances scientifiques en publiant et participe à l'enrichissement de sa propre discipline. En tant qu'enseignant, il transmet les connaissances récentes issues des recherches conduites dans son domaine de spécialisation aux étudiants ». Le modèle pédagogique dominant est gouverné par l'idée d'une transmission verticale, les doctorants s'exprimant dans un registre «maître-élève " par ce qu'ils sont et ce qu'ils savent: «Ce fut très intéressant, j’ai aimé entrer en relation avec les étudiants, leur transmettre des connaissances, et surtout les amener à se questionner, à réfléchir, à exercer leur esprit critique. J'ai vraiment essayé d'être à l'écoute, disponible, attentif, bienveillant envers tous les étudiants; j'ai beaucoup préparé mes cours, avec beaucoup d'intérêt, pour transmettre au mieux ce qui me semblait essentiel aux étudiants. Et les étudiants me l'ont bien rendu: les cours se sont très bien passés, j'ai pu voir certains étudiants progresser véritablement et s'épanouir dans la discipline enseignée, j'ai eu 
plusieurs retours positifs, ce qui m'a réjoui. Certains m'ont dit vouloir poursuivre leurs études en master suite à mes enseignements ; cela me satisfait grandement. »

\subsubsection{L'enseignant-chercheur entrepreneur}

«L'université française a beau rester une bureaucratie d'État, elle est devenue un monde d'entrepreneurs" (Dubet, 2003, p. 377). Si François Dubet soulignait ainsi en 2003 que "chaque universitaire est plus ou moins l'entrepreneur de sa propre carrière », les répondants estiment que le métier d'enseignant-chercheur a évolué ou s'est transformé au cours des dix dernières années avec des mesures législatives et une baisse des financements publics qui obligent les enseignants-chercheurs à rechercher de nouvelles sources de financement. Un doctorant indique : «Les enseignants-chercheurs tendent à devenir des entreprises de soi-même... le néolibéralisme ne privilégie personne les chercheurs en sont aussi les sujets! Un changement dans le rapport au savoir; les autorités constituées avec les statuts etc. n'ont plus belle presse, la culture du si "petit monde" change peut-être en ce sens... plus de femmes sont présentes... toujours peu de minorités... » Cet extrait illustre le sentiment d'une dévalorisation du métier dans sa dimension scientifique : «Il me semble que les enseignants-chercheurs doivent de plus en plus diversifier leurs activités, savoir se vendre, se battre pour décrocher des financements et pouvoir engager leurs recherches. Cela est caractéristique de la société actuelle, hypermoderne, hyper-libérale, centrée sur le rendement économico-financier... l'universitaire, à l'origine, visait à acquérir puis à transmettre un "savoir universel"... j'ai l'impression que depuis quelques années la quantité l'emporte sur la qualité. Il n'est pas possible d'être sur tous les fronts et de produire des travaux de recherche de qualité. Il me semble que les activités de recherche en pâtissent grandement ; je connais un certain nombre d'enseignants-chercheurs qui ne trouvent plus le temps... de chercher! Cela explique-t-il le fait que certains enseignants-chercheurs essaient de trouver des moyens permettant de limiter leurs multiples activités pour se recentrer sur leurs travaux de recherche, et ainsi avoir un peu plus de temps pour penser? » Il s'agit là de la pression de la «publication », pour " rapporter des "points" pour le laboratoire ». In fine, malgré ces réponses et ces écrits, le projet professionnel initial des doctorants ne s'est pas modifié pour 57,1 \% d'entre les répondants, depuis l'inscription en thèse de doctorat.

\section{Interprétation des résultats}

Le parcours des doctorants qui ont répondu au questionnaire peut être comparé à une forme d'engagement professionnel, c'est-à-dire :

L'ensemble dynamique des comportements qui, dans un contexte donné, manifeste l'attachement à la profession, les efforts consentis pour elle ainsi que le sentiment du devoir vis-à-vis d'elle et qui donne sens à la vie professionnelle au point de marquer l'identité personnelle et professionnelle (De Ketele dans Jorro \& De Ketele, 2013, p. 11).

21 Or, une telle attitude peut sembler surprenante car ces doctorants ne sont pas encore entrés dans le métier et n'ont pas même la certitude de pouvoir y prétendre. Si nous revenons aux deux axes heuristiques qui ont orienté notre recherche, le rôle des pairs et l'influence de la matrice disciplinaire dans les parcours et les vécus des doctorants, nous pouvons indiquer que l'appui des pairs et les relations interprofessionnelles constituent des éléments essentiels de la socialisation de ces doctorants. Ces premiers résultats 
montrent que l'influence des pairs sur les doctorants agit non seulement sur leur manière d'enseigner mais aussi sur l'appropriation de la culture du milieu. Quant à l'influence de la matrice disciplinaire, notre échantillonnage, non représentatif, ne nous a pas permis pas d'établir un lien précis entre la discipline d'appartenance et les modalités de socialisation professionnelle, ce même si nous estimons qu'elles sont dépendantes. Nous notons le rapport particulier qu'entretiennent les doctorants avec l'activité pédagogique.

\subsection{Devenir doctorant, un engagement sur le long terme}

Nous notons que les motifs de l'inscription en thèse de doctorat appartiennent au registre de l'engagement: "L'envie de "faire de la recherche". J'ai travaillé dix ans dans l'enseignement primaire et j'avais envie de passer à autre chose. Ou du moins de tenter. Indépendamment de la suite, j'envisage le doctorat comme une aventure scientifique, une expérience nouvelle... » Pour certains étudiants, ce projet s'est constitué dès la licence ou la maitrise : «Le souhait de réaliser une thèse de doctorat était clairement présent depuis la licence. La thématique sur laquelle je travaille actuellement était déjà largement présente dans mon esprit, au cœur de mes questionnements et préoccupations. Motivé pour poursuivre mes études en master recherche puis en doctorat, j'ai changé d'université pour m'inscrire dans une université dont les axes de recherche du laboratoire étaient tout indiqués pour m'accueillir.»

\subsection{L'influence des pairs}

23 Les échanges informels entre les titulaires, maîtres de conférences et les doctorants, représentent des sources d'information et de formation au métier d'enseignantchercheur. Les maîtres de conférences aident ou fournissent des supports pour la préparation des cours et l'encadrement des étudiants. Cette dimension est omniprésente dans les réponses : «Avec le maître de conférence responsable du cours, on échange sur les difficultés rencontrées dans le cours magistral, on réfléchit à la façon de présenter les concepts pour les faire comprendre de façon informelle. » Le directeur de thèse est aussi présenté comme un initiateur pour les premiers pas dans l'enseignement : «J'ai la chance de pouvoir poser des questions, demander conseil à certaines personnes ressources dans le département comme l'enseignante responsable pédagogique de la licence ou encore ma directrice de thèse. "

Les pairs semblent avoir été à l'origine d'un compagnonnage, dans l'esprit d'une transmission qui mène à une forme de reproduction des pratiques d'enseignement principalement. Mais cela semble aussi participer d'une socialisation, d'une appropriation des caractéristiques sociales de la profession.

\section{Conclusion : une identité professionnelle en construction}

Les premières conclusions de notre enquête rejoignent celles qui ont été établies par Saeed Paivandi (2010) dans sa recherche sur l'expérience pédagogique des moniteurs: «l'association entre recherche et enseignement est très largement plébiscitée » et «la relation avec les autres enseignants constitue un facteur déterminant de l'insertion des 
moniteurs en tant qu'enseignants débutants ». La reproduction des pratiques des aînés paraît être la première approche de l'activité d'enseignement. La qualité de l'enseignement est d'abord définie par l'excellence académique et l'articulation entre enseignement et recherche. Cet attachement à des formes de traditions pédagogiques et aux conseils des aînés nous interroge sur les conditions de l'autonomisation de ces débutants pour élaborer leurs propres choix pédagogiques dans un cadre malgré tout contraint.

En outre, bien que n'étant pas investis des mêmes missions que les titulaires, en particulier pour l'engagement dans des activités d'administration de l'enseignement ou de la recherche, les doctorants semblent reproduire les discours sur le métier des maîtres de conférences ou des professeurs. En particulier, ils ressentent une rupture entre « un monde universitaire" considéré comme "une communauté d'idées et de valeurs universelles » (Paivandi, 2010) et un monde universitaire dépendant du marché et de la concurrence. Au terme de cette étude exploratoire, des perspectives d'approfondissement se dessinent. Il s'agirait de saisir les éléments à travers lesquels des doctorants, qui exercent une mission d'enseignement, construisent une carrière académique en se développant professionnellement dans leur contexte tout en s'appropriant l'héritage de leur milieu. Une telle approche nécessiterait de conduire plusieurs entretiens sur un temps long et sur les thèmes suivants : leurs représentations des changements structurels contemporains, de leurs relations avec leurs pairs, de leurs pratiques pédagogiques selon leurs approches disciplinaires et les publics d'étudiants auxquels ils s'adressent. L'enjeu serait de repérer d'éventuelles mutations identitaires à l'œuvre en comparant leurs discours avec ceux de leurs aînés.

\section{BIBLIOGRAPHIE}

Adé, D. \& Piot, T. (2018). La formation entre universitarisation et professionnalisation. Tensions et perspectives dans des métiers de l'interaction humaine. Rouen : Presses universitaires de Rouen et du Havre.

Bertrand, C. (2014). Soutenir la transformation pédagogique dans l'enseignement supérieur, Rapport à la demande de Mme Simone Bonnafous, directrice générale pour l'enseignement supérieur et l'insertion professionnelle. Paris : Ministère de l'Éducation nationale, de l'Enseignement supérieur et de la Recherche.

Bourdieu, P. (1984). Homo Academicus. Paris : Éditions de Minuit.

Dahan, A. \& Mangematin, V. (2010). Recherche, ou temps perdu ? Vers une intégration des tâches administratives au métier d'enseignant-chercheur. Annales des Mines - Gérer et comprendre, 4(102), 14-24.

De Ketele, J.-M. (2013). L'engagement professionnel : tentatives de clarification conceptuelle. Dans A. Jorro et J.-M. De Ketele (éds.), L'engagement professionnel en éducation et en formation (pp. 7-21). Bruxelles : De Boeck. 
Dubar, C. (1998). La Socialisation. Construction des identités sociales et professionnelles. Paris : Armand Colin.

Dubet, F. (2003). Problèmes d'une sociologie de l'enseignement supérieur. Dans G. Felouzis (éd.), Les mutations actuelles de l'Université (pp. 363-380). Paris : PUF.

Espéret, E. (2001). Nouvelle définition des tâches des enseignants et des enseignants-chercheurs dans l'enseignement supérieur français. Paris : Ministère de l'Éducation nationale.

Etienne, R. \& Annoot, E. (2014). L'entrée dans le métier d'enseignant-chercheur. Communication présentée lors du colloque de l'AIPU, Mons. Repéré à http://hosting.umons.ac.be/php/ AIPU2014/DOC/AIPU2014_livret-resume.pdf

Faure, S., Soulié, C. \& Millet, M. (2006). Rationalisation, bureaucratisation et mise en crise de l'ethos académique, Regards sociologiques, 31, 107-140.

Faure, S., Soulié, C. \& Millet, M. (2008). Visions et divisions de l'université, vers la fin du métier d'enseignant-chercheur ? Recherche et formation, 57, 79-87.

Fave-Bonnet, M.-F. (2002). Conflits de missions et conflits de valeurs : la profession universitaire sous tension. Connexions, 78, 31-45.

Lameul, G. (2008). Les effets de l'usage des technologies d'information et de communication en formation d'enseignants, sur la construction des postures professionnelles, Savoirs, 2(17), 71-94.

Millet, C. (2011). Parcours professionnels et genre : les carrières des enseignants-chercheurs. Dans A.-M. Vonthron, S. Pohl et P. Desrumeaux (éds.), Développement des identités, des compétences et des pratiques professionnelles (pp. 43-54). Paris : L'Harmattan.

Millet, C., Oget, D. \& Sonntag, M. (2016). Analyse du discours des enseignants-chercheurs sur leur activité professionnelle : vers une transformation identitaire du métier ?, Phronesis, 4(4), 56-63, Sherbrooke : Éditions du Champ social. DOI : 10.7202/1036708ar

Paivandi, S. (2010). L'expérience pédagogique des moniteurs comme analyseur de l'université, Revue française de pédagogie, 172. DOI : 10.4000/rfp.2200

Paul, M. (2009). Accompagnement, Recherche et formation, 62. DOI : 10.4000/

rechercheformation.435

Pratt, M.G., Rockmann, K.W. \& Kaufmann, J.B. (2006). Constructing professional identity: The role of work and identity learning cycles in the customization of identity among medical residents, Academy of Management Journal, 49(2), 235-262.

Rege Colet, N. \& Berthiaume, D. (2009). Savoir ou être ? Savoirs et identités professionnels chez les enseignants universitaires. Dans R. Hofstetter et B. Schneuwly (dir.), Savoirs en (trans)formation au cœur des professions de l'enseignement et de la formation (pp. 137-162). Bruxelles : De Boeck.

Serre, D. (2015). Être doctorant-e. Socialisations, contextes, trajectoires, Socio-logos, 10. Repéré à http://socio-logos.revues.org/2924

\section{NOTES}

1. Le programme a été porté par David Adé au laboratoire CETAPS (université de Rouen) en coopération scientifique avec Thierry Piot du laboratoire CERSE devenu CIRNEF (université de Caen) et financé par la Région Haute-Normandie dans le cadre du Grand réseau de recherche «Culture et société en Normandie» (GRR CSN). Richard Etienne, Emmanuelle Annoot et Paule Biaudet sont les auteurs d'un chapitre dans l'ouvrage La formation entre universitarisation et 
professionnalisation. Tensions et perspectives dans des métiers de l'interaction humaine, intitulé : «Les enseignants-chercheurs débutants en France : l'urgence de la pédagogie ».

2. Cette recherche a fait l'objet d'une communication orale autour de ses premiers résultats lors du colloque intitulé : Sortir de la confusion des savoirs et des valeurs par la différenciation des domaines du symbolique et des textes, porté par la professeure Marie-Louise Martinez (laboratoire CIRNEF, EA7454), qui s'est déroulé à l'université de Rouen en mai 2016.

\section{RÉSUMÉS}

Cet article propose une réflexion sur les transformations qui façonnent l'enseignement supérieur français ces dernières années, à travers le cas spécifique des doctorants découvrant le métier d'enseignant-chercheur. Nous proposons d'accéder aux représentations du métier qu'ont ces doctorants en mission d'enseignement à partir d'un recueil de données effectué par un questionnaire distribué auprès de quarante et un étudiants doctorants en sciences humaines et sociales, droit économie gestion, sciences santé, lettres, langues. Précisément, nous analysons le lien entre deux composantes fondamentales du métier : l'enseignement et la recherche. À l'heure de l'autonomie des universités, nos résultats mettent en avant chez ces débutants une tendance à la reproduction des pratiques et l'adhésion à la culture professionnelle de leurs aînés. Ces premières conclusions nous enjoignent à une interrogation sur l'identité professionnelle actuelle des universitaires français et à poursuivre les recherches sur l'entrée dans un métier qui se transforme, en fédérant les recherches sur le domaine.

This article proposes a discussion on transformations that outline French higher education system in recent years, through the specific case of $\mathrm{PhD}$ students discovering the academic profession. We focus on the representations of these doctoral students with teaching missions from data collected by a questionnaire distributed to $41 \mathrm{PhD}$ students in the human and social sciences, law and economic management, health sciences, literature and languages. Precisely, we analyze the link between two fundamental components of the profession: teaching and research. At the time of university autonomy, our results put forward a tendency for these beginners to the reproduction of the practices and the adhesion to the professional culture of their elders. These first conclusions enjoin us to an interrogation on the current professional identity of French academics and to pursue research on entry into a profession that is transformed, by federating research on the field.

\section{INDEX}

Mots-clés : doctorants, enseignant-chercheur, représentations, identité professionnelle

Keywords : PhD students, academic teacher-researcher, representation, professional identity 


\section{AUTEURS}

\section{CHARLYNE MILLET}

Docteure en sciences de l'éducation de l'université de Strasbourg, chercheure associée à l'université du Havre Normandie, UMR CNRS IDEES-Le Havre, Normandie Univ, UNIHAVRE, IDEES, 76600 Le Havre, France

\section{EMMANUELLE ANNOOT}

Professeure en sciences de l'éducation à l'université de Rouen Normandie, Normandie Univ, UNIROUEN, CIRNEF, 76000 Rouen, France 